\title{
ON EXPLOSIONS OF SOLUTIONS TO A SYSTEM OF PARTIAL DIFFERENTIAL EQUATIONS MODELLING CHEMOTAXIS
}

\author{
W. JÄGER AND S. LUCKHAUS
}

\begin{abstract}
A system of partial differential equations modelling chemotactic aggregation is analysed (Keller-Segel model). Conditions on the system of parameters are given implying global existence of smooth solutions. In two space dimensions and radially symmetric situations, explosion of the bacteria concentration in finite time is shown for a class of initial values.
\end{abstract}

\section{INTRODUCTION}

The aggregation of organisms sensitive to a gradient of a chemical substance has been of great interest to biologists and mathematicians, trying to model and to simulate the observed pattern formation. The most familiar example of a species showing chemotactic movement is dictyostelium discoideum [G] Model equations were set up and analysed e.g. by Keller and Segel [K-S], W. Alt [A1, A2], and R. Schaaf [S]. The following model was introduced by Keller and Segel to describe the dynamics of a population (concentration $u$ ) moving in a domain $\Omega$ and following a gradient of a chemotactic agens (concentration $v$ ) produced by the population itself,

$$
\begin{aligned}
& \partial_{t} u=\Delta u-\chi \nabla(u \nabla v) \quad \text { in } \Omega, \\
& \partial_{t} v=\gamma \Delta v-\mu v+\beta u, \\
& u(0, \cdot)=u_{0}, \quad v(0, \cdot)=v_{0}, \quad u_{0}, v_{0} \geq 0 \\
& \partial_{\nu} u(t, \cdot)=\partial_{\nu} v(t, \cdot)=0 \text { on } \partial \Omega .
\end{aligned}
$$

$\chi, \gamma, \mu, \beta$ are positive constants. Due to the experimental facts the diffusion coefficient of the substance $v$ is assumed to be large, of order $\frac{1}{\varepsilon}, \varepsilon$ small, and $\beta=\gamma \alpha$ where $\alpha$ and $\mu$ are of order 1 . From equation (1) we obtain

$$
\bar{u}(t)=\overline{u_{0}}, \quad \frac{1}{\gamma}\left(\partial_{t}-\mu\right) \bar{v}=\alpha \bar{u}=\alpha \overline{u_{0}},
$$

where $\bar{w}$ denotes $(1 /|\Omega|) \int_{\Omega} w d x$. Therefore, if we consider $\tilde{v}:=v-\bar{v}$ we get $\frac{1}{\gamma}\left(\partial_{t}-\mu\right) \tilde{v}=\Delta \tilde{v}-\alpha\left(u-\overline{u_{0}}\right)$. Hence, for small $\varepsilon$ we may consider the system

$$
\partial_{t} u=\Delta u-\chi \nabla(u \nabla v), \quad 0=\Delta v-\alpha\left(u-\overline{u_{0}}\right) .
$$

Received by the editors December 7, 1989.

1980 Mathematics Subject Classification (1985 Revision). Primary 35B05, 35B30, 35B35, 35B65; Secondary 92A15, 92A17.

This work has been supported by the Deutsche Forschungsgemeinschaft (SFB: Stochastische Mathematische Modelle). 
Rescaling

leads to the system

$$
v^{*}=\frac{v}{\alpha \overline{u_{0}}}, \quad u^{*}=\frac{u}{\overline{u_{0}}}
$$

$$
\partial_{t} u^{*}=\Delta u^{*}-\chi^{*} \nabla\left(u^{*} \nabla v^{*}\right), \quad 0=\Delta v^{*}-\left(u^{*}-1\right), \quad \chi^{*}=\alpha \overline{u_{0}} \chi .
$$

In the following we will drop the ${ }^{*}$ on $u$ and $v$. By the maximum principle we immediately obtain that for any solution $(u, v)$ we have $u \geq 0$. $v$ can be directly computed if we know $u$.

We shall study the dynamic behavior of this approximating system and show that for small $\chi^{*}$ there exists a unique smooth global positive solution $u$ if the initial datas are smooth. For large $\chi^{*}$ and $n \geq 2$ there exist solutions $u$ which explode in finite time. Explosion in chemotaxis has been observed by Childress [C]. In this behavior the model equations are showing an effect similar to the formation of fruiting bodies observed in colonies of chemotactic microorganisms $[\mathrm{G}]$.

\section{Mathematical Results}

We assume that $\Omega$ is a bounded open set in $\mathbf{R}^{2}, \partial \Omega$ is a $C^{1}$-boundary, $u_{0}$ is $C^{1}$ and satisfies the boundary condition.

Theorem. (a) There exists a critical number $c(\Omega)$ such that $\alpha \cdot \overline{u_{0}} \cdot \chi<c(\Omega)$ implies that there exists a unique, smooth positive solution to (3) for all time.

(b) Let $\Omega$ be a disk. There exists a positive number $c^{*}$ with the following property: If $\alpha \cdot \overline{u_{0}} \chi>c^{*}$ then radially symmetric positive initial values can be constructed such that explosion of $u$ happens in the center of the disc in finite time.

More exactly, the following statement, implying (a), holds.

Proposition. Let $\Omega$ be a domain satisfying the smoothness assumptions.

Let $u$ be a smooth positive solution to (3) and $t^{*}$ the maximal time of existence, $0<t^{*} \leq+\infty$. There exists a positive number $c_{1}(\Omega)$ such that $t^{*}<\infty$ implies

$$
\lim _{k \rightarrow \infty} \varlimsup_{t \uparrow t^{*}} \chi^{*} \int_{\Omega}(u-k)_{+} d x \geq c_{1}(\Omega)
$$

Remarks. (i)(a) contains information on the rate of explosion if it happens in finite time.

(ii) It would be interesting to know more about the set of explosion points at $t^{*}$. The solution may globally exist as weak solutions. The development of singularities after a finite time $t^{*}$ is another important topic to be studied.

(iii) Global existence of smooth solutions to systems similar to (1) was studied recently by Pozio and Tesei [P-T].

\section{A PRIORI ESTIMATES}

We are going to prove the proposition (a) concerning smooth solutions for small time. Consider the test function $\varphi=(u-k)_{+}^{m-1}$ where $k \geq 0, m>1$, and multiply the system

$$
\partial_{t} u-\Delta u+\chi^{*} \nabla(u \nabla v)=0, \quad-\Delta v-(u-1)=0
$$


by $\varphi$ and integrate over $\Omega$. Partial integration leads to

$$
\begin{aligned}
0= & \frac{d}{d t} \frac{1}{m} \int_{\Omega}(u-k)_{+}^{m} d x+4 \frac{m-1}{m^{2}} \int_{\Omega}\left|\nabla(u-k)_{+}^{m / 2}\right|^{2} d x \\
& -\chi^{*} \int_{\Omega} \nabla v \cdot \nabla\left(\frac{m-1}{m}(u-k)_{+}^{m}+k(u-k)_{+}^{m-1}\right) d x .
\end{aligned}
$$

We use the second equation of (5) in order to compute the last integral $I$. Collecting the proper terms we get

$$
\begin{aligned}
I= & \frac{m-1}{m} \int_{\Omega}(u-k)_{+}^{m} d x+k \int_{\Omega}(u-k)_{+}^{m-1} d x-\frac{m-1}{m} \int_{\Omega}(u-k)_{+}^{m+1} d x \\
& -\frac{2 m-1}{m} \int_{\Omega}(u-k)_{+}^{m} d x-k^{2} \int_{\Omega}(u-k)_{+}^{m-1} d x .
\end{aligned}
$$

Altogether, we obtain

$$
\begin{aligned}
\frac{d}{d t} \frac{1}{m} \int_{\Omega}(u-k)_{+}^{m} d x+4 \frac{m-1}{m^{2}} \int_{\Omega}\left|\nabla(u-k)_{+}^{m / 2}\right|^{2} d x \\
-\chi^{*} \frac{m-1}{m} \int_{\Omega}(u-k)_{+}^{m+1} d x \\
=\chi^{*}\left(\frac{2 m-1}{m} k-\frac{m-1}{m}\right) \int_{\Omega}(u-k)_{+}^{m} d x \\
\quad+\chi^{*} k(k-1) \int_{\Omega}(u-k)_{+}^{m-1} d x .
\end{aligned}
$$

The integral with power $m+1$ has to be estimated. To this purpose we may choose $k$ large enough such that measure $\left\{x \mid(u-k)_{+}(x)=0\right\}>\frac{1}{2}|\Omega|$. We apply the Sobolev inequality in the case $n=2$,

$$
\int_{\Omega} \varphi^{2} d x \leq c_{1}(\Omega)\left(\int_{\Omega}|\nabla \varphi| d x\right)^{2}
$$

and obtain

$$
\begin{aligned}
\int(u & -k)_{+}^{m+1} \leq c_{1}(\Omega)\left(\int\left|\nabla(u-k)_{+}^{(m+1) / 2}\right| d x\right)^{2} \\
& \leq c_{1}(\Omega)\left(\frac{m+1}{m}\right)^{2} \int_{\Omega}(u-k)_{+} d x \int_{\Omega}\left|\nabla(u-k)_{+}^{m / 2}\right|^{2} d x .
\end{aligned}
$$

Setting $(u-k)_{+}^{m / 2}=: w$, we have, using the Cauchy-Schwarz inequality,

$$
\left|\nabla(u-k)_{+}^{(m+1) / 2}\right|=\left|\nabla w^{1+1 / m}\right|=\frac{m+1}{m}\left|w^{1 / m}\right| \cdot|\nabla w| .
$$

The inequality implies

$$
\begin{aligned}
4 \frac{m-1}{m^{2}} \int_{\Omega}\left|\nabla(u-k)_{+}^{m / 2}\right|^{2} d x-\chi^{*} \frac{m-1}{m} \int_{\Omega}(u-k)_{+}^{m+1} d x \\
\geq \\
\quad\left\{4 \frac{m-1}{m^{2}}-\frac{m-1}{m}\left(\frac{m+1}{m}\right)^{2} c_{1}(\Omega) \chi^{*} \int_{\Omega}(u-k)_{+} d x\right\} \\
\quad \times \int_{\Omega}\left|\nabla(u-k)_{+}^{m / 2}\right|^{2} d x
\end{aligned}
$$


If the inequality (4) does not hold, we could estimate the bracket \{\} by a positive number for $t$ close to $t^{*}$ and $k$ large, $t \geq t_{0}, k \geq k_{0}$. Thus we would get from (6),

$$
\frac{d}{d t} \int_{\Omega}(u-k)_{+}^{m} d x \leq c_{2}(\Omega, k)+c_{3}(k) \int_{\Omega}(u-k)_{+}^{m} d x \quad \text { for } t \geq t_{1}
$$

and therefore finally obtain a bound for the $L^{m}$-norms of the solution.

Using the equations (5) and standard arguments for elliptic and parabolic equation, one can control all Sobolev-norms needed to show smoothness for all time.

\section{BLOW UP OF RADIALLY SYMMETRIC SOLUTIONS}

We now prove part (b) of the theorem. The main tool is the construction of the lower solutions, but not to the system directly. We restrict ourselves to radially symmetric case and remark that radially symmetric smooth initial datas imply the local existence of smooth symmetric solutions.

$$
U(t, \rho):=\int_{0}^{\sqrt{\rho}}(u(t, r)-1) r d r, \quad r=|x|, 0 \leq \rho \leq R .
$$

Integrating the first equation of (5) over $B_{\sqrt{\rho}}=\{|x|<\sqrt{\rho}\}$, we obtain for each term,

$$
\begin{gathered}
\int_{B \sqrt{\rho}} \partial_{t} u d x=2 \pi \partial_{t} U(t, \rho), \\
-\int_{B \sqrt{\rho}} \Delta u d x=2 \pi \sqrt{\rho} \partial_{\sqrt{\rho}} u(t, \sqrt{\rho}) \\
=2 \pi \sqrt{\rho} \partial_{\sqrt{\rho}}\left(\frac{1}{\sqrt{\rho}} \partial_{\sqrt{\rho}}\right) U(t, \rho)=8 \pi \rho \partial_{\rho}^{2} U(t, \rho), \\
\int_{B \sqrt{\rho}} \nabla(u \nabla v) d x=2 \pi \sqrt{\rho} u \partial_{\sqrt{\rho}} v \\
=\left(2 \partial_{\rho} U+1\right) \int_{B \sqrt{\rho}} \Delta v d x=-2 \pi\left(\partial_{\rho} U^{2}+U\right),
\end{gathered}
$$

and therefore

$$
\partial_{t} U-4 \rho \partial_{\rho}^{2} U-\chi^{*} \partial_{\rho} U^{2}-\chi^{*} U=0 .
$$

$U$ satisfies the following initial-boundary conditions

$$
U(0, \rho)=\int_{0}^{\sqrt{\rho}}\left(u_{0}-1\right) r d r, \quad U(t, 0)=U(t, R)=0 .
$$

We may assume that the initial values $u_{0}$ are such that $U(0, \cdot)>0$ in $] 0, R[$. This can be obtained by a positive function monotonely decreasing with respect to $r$ and sufficiently large at the origin. See the Figure.

We now construct a subsolution $W$ satisfying

$$
\begin{gathered}
\partial_{t} W-4 \rho \partial_{\rho}^{2} W-\chi^{*} \partial_{\rho} W^{2}-\chi^{*} W \leq 0, \\
W(0, \rho) \leq U(0, \rho), \quad W(t, 0)=W(t, R)=0
\end{gathered}
$$




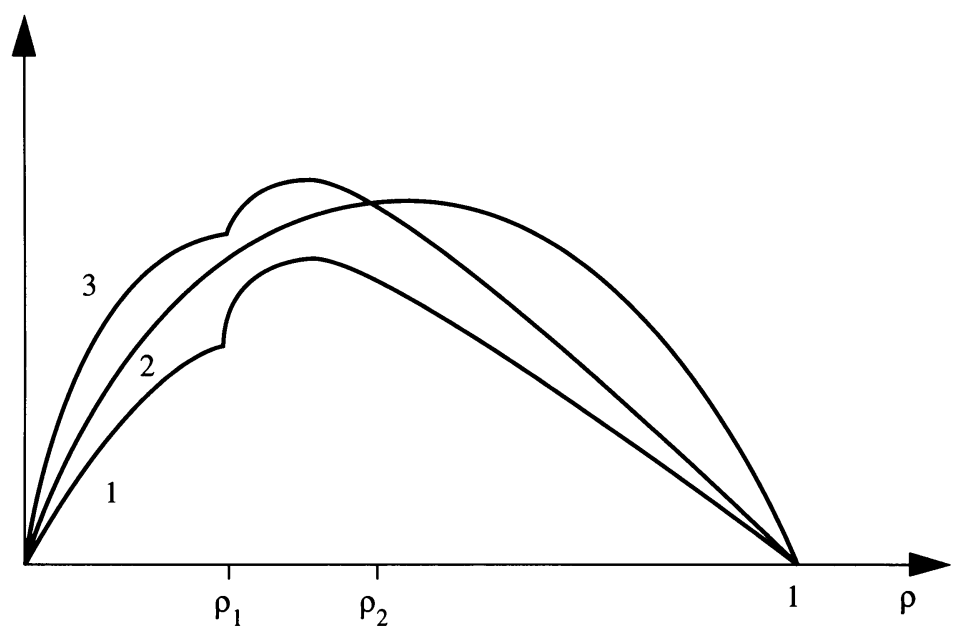

FIGURE

1,3 Graphs of subsolution $W$ at time $t_{0}$ and time $t_{1}<\bar{t}$.

2 Graph of solution $U$ at time $t_{0}$.

and therefore $W(t, \rho) \leq U(t, \rho)$ for all $t, \rho$ considered. We shall produce a weak subsolution up to time $\bar{t}$, such that

$$
\lim _{t \rightarrow \bar{t}} \sup _{\rho<\varepsilon} W(t, \rho) \geq \omega>0
$$

for each $\varepsilon>0$. This will mean blow up must have occurred at a time $t^{*} \leq \bar{t}$ in the center of the disk.

Choose parameters $0<\rho_{1}<\rho_{2}<1$, and define

$$
W(t, \rho)= \begin{cases}\frac{a \rho}{\rho+\tau^{3}} & \text { for } \rho<\rho_{1}, \\ \gamma\left(1-\rho-\frac{\left(\rho_{2}-\rho\right)_{+}^{2}}{\rho_{2}}\right) & \text { for } \rho_{1} \leq \rho,\end{cases}
$$

where

$$
\tau=\rho_{0}-b t, \quad \gamma=\left(1-\rho_{1}-\frac{\left(\rho_{2}-\rho_{1}\right)^{2}}{\rho_{2}}\right)^{-1} \frac{a \rho_{1}}{\rho_{1}+\tau^{3}},
$$

$a, \rho_{0}, b$ are parameters to be chosen such that (9) holds. Obviously (10) is satisfied by this $W$. For $\rho<\rho_{1}$ we obtain

$$
\begin{aligned}
\partial_{t} W & =\frac{3 b \tau^{2}}{\rho+\tau^{3}} \cdot W, \quad \partial_{\rho} W=\frac{\tau^{3} a}{\left(\rho+\tau^{3}\right)^{2}}, \quad \rho \partial_{\rho}^{2} W=-\frac{2 \tau^{3}}{\left(\rho+\tau^{3}\right)^{2}} W, \\
\partial_{t} W & -4 \rho \partial_{\rho}^{2} W-2 \chi^{*} W \partial_{\rho} W-\chi^{*} W \\
& =\left\{\frac{3 b \tau^{2}}{\rho+\tau^{3}}+\left(8-2 a \chi^{*}\right) \frac{\tau^{3}}{\left(\rho+\tau^{3}\right)^{2}}-\chi^{*}\right\} W<0
\end{aligned}
$$

for $0<\rho<1$, if

$$
a \chi^{*}-4>0 \text { and } b<\frac{1}{3} \sqrt{\frac{\left(2-a \chi^{*}-8\right) \chi^{*}}{\tau^{*}}}
$$


For $\rho>\rho_{1}$ we compute

$$
\begin{gathered}
\partial_{t} W=\frac{\gamma^{\prime}}{\gamma} W=\frac{2 b \tau^{2}}{\rho_{1}+\tau^{3}} W, \quad \partial_{\rho} W=-\gamma(t)\left(1-\frac{2\left(\rho_{2}-\rho\right)_{+}}{\rho_{2}}\right), \\
\rho \partial_{\rho}^{2} W=\left\{\begin{array}{lc}
\frac{-2 \gamma(t)}{\rho_{2}} & \text { for } \rho<\rho_{2}, \\
0 & \text { for } \rho \geq \rho_{2}, \\
\partial_{t} W-4 \rho \partial_{\rho}^{2} W-2 \chi^{*} W \partial_{\rho} W-\chi^{*} W \\
\leq\left\{\frac{2 b \rho_{0}^{2}}{\rho_{1}}+\frac{8}{\rho_{2}\left(1-\rho_{2}\right)}-\chi^{*}\left(1-\frac{2 a}{1-\rho_{2}}\right)\right\} W<0
\end{array}\right.
\end{gathered}
$$

if $a<\frac{1}{2}\left(1-\rho_{2}\right), \chi^{*}>8 / \rho_{2}\left(1-\rho_{2}-2 a\right)$ and $b \rho_{0}^{2}$ sufficiently small.

In $\rho=\rho_{1}$, the first derivative with respect to $\rho$ has a positive jump and therefore the correct sign. Assume that $U(0, \cdot)$ is strictly positive in $] 0,1[$. Since

$$
\partial_{\rho} U(0,0)=\frac{1}{2}\left(u_{0}(0)-1\right)>0, \quad \partial_{\rho} U(0,1)=\frac{1}{2}\left(u_{0}(0)-1\right)<0
$$

by assumption and

$$
\partial_{\rho} W(0,0)=a / \rho_{0}^{3}, \quad \partial_{\rho} W(0,1)=-\gamma(0),
$$

we can $\rho_{0}$ choose large enough in order to obtain $W(0, \cdot) \leq U(0, \cdot)$. Thus, the comparison is possible. The explosion time is bounded by $\rho_{0} / b$.

\section{REFERENCES}

[A1] W. Alt, Orientation of cells migrating in a chemotactic gradient, Lecture Notes in Biomath., vol. 38, Springer-Verlag, 1980, pp. 353-366.

[A2] _ Biased random walk models for chemotaxis and related diffusion approximations, $\mathrm{J}$. Math. Biol. 9 (1980), 147-177.

[C] S. Childress, Chemotactic collapse in two dimensions, Lecture Notes in Biomath., vol. 55, Springer, 1984, pp. 61-68.

[G] G. Gerisch et al., Philos. Trans. Roy. Soc. London Ser. B 272 (1975), 181-192.

[K-S] E. F. Keller and L. A. Segel, J. Theoret. Biol. 26 (1970),

[K] E. F. Keller, Assessing the Keller-Segel model: How has it fared, Lecture Notes in Biomath., vol. 38, Springer-Verlag, 1980, pp. 379-387.

[P-T] M. A. Pozio and A. Tesei, Global existence of solutions for a strongly coupled parabolic system, Preprint, Istituto per le Applicatione del Calcolo "Mauro Picone", Roma, 1988.

[S] R. Schaaf, Global branches of one dimensional stationary solutions to chemotaxis systems and stability, Lecture Notes in Biomath., vol. 55, Springer, 1984, pp. 341-349.

SONDERFoRSCHUNGSBereich 123, UNiversitÄt Heidelberg, IM NeUENheimer Feld 294, W6900 HeIDELBERG, GeRMANY

Institut für ANgewandte Mathematik, Universität Bonn, Wegeler Strasse 6, W-5300 BONN 1, GERMANY 